\title{
Discharge of a 2D magnetic silo
}

\author{
Louison Thorens ${ }^{1,2, *}$, Maud Viallet ${ }^{1}$, Knut Jørgen Måløy ${ }^{2}$, Mickaël Bourgoin $^{1}$, and Stéphane Santucci ${ }^{1,3}$ \\ ${ }^{1}$ Univ Lyon, ENS de Lyon, Univ Claude Bernard, CNRS, Laboratoire de Physique, F-69342 Lyon, France \\ ${ }^{2}$ PoreLab, The Njord Centre, Department of Physics, University of Oslo, P. O. Box 1048 Blindern, N-0316 Oslo, Norway \\ ${ }^{3}$ Lavrentyev Institute of Hydrodynamics, Siberian Branch of the Russian Academy of Sciences, Novosibirsk, Russia
}

\begin{abstract}
We investigate experimentally the discharge of a 2D-silo, a Hele-Shaw cell, filled with a mono layer of ferromagnetic grains submitted to an external magnetic field $\mathbf{B}$ perpendicular to the cell plane. In this case the magnetic pair interactions are repulsive. We show that the granular flow rate decreases systematically with the amplitude of the external magnetic field applied. Interestingly, while the output flow rate remains constant during an experiment, we reveal very large spatio-temporal fluctuations of the packing density within the cell, particularly evident for magnetic field of high amplitudes.
\end{abstract}

\section{Introduction}

Granular materials have been, over the last decades, celebrated for their particular static and dynamic behaviour [1-3]. One of the most daily encountered granular system is certainly the hourglass where grains flow from a top vessel to a lower one driven only by gravity. The first model to describe the flow of particles flowing through a small aperture was given by Beverloo in 1961 [4]. The particles flow rate is supposed constant and the only size of interest is the opening aperture $w$ which leads to a drainage velocity $v \sim \sqrt{g w}$. Since the opening surface is proportional to the opening width $w$ in a 2D geometry, the output mass flow rate $q$ is then proportional to $\rho \sqrt{g} w^{3 / 2}$ with $\rho$ the grains density. Beverloo's law gives a better fit to the experimental observation with the following expression: $q=C \rho \sqrt{g}\left(w-w_{m}\right)^{3 / 2}$ where $C$ is a constant and $w_{m}$ an opening width correction of about 1 to 2 grain diameters. The grain flow rate is observed to be constant and useful compared to a clepsydra, but the justification of this interesting property is still under debate. The Janssen effect is commonly proposed to justify that the flow rate given by the Beverloo law is independent of the height of the granular column. However, several experimental measurements either in velocity driven [5] or gravity driven conditions $[6,7]$ have shown that such argument is improper. A clear understanding of the physical origin of the Beverloo law still remains to be unveiled.

Recently, thanks to the use of ferromagnetic particles, we could demonstrate the emergence of a tunable "Magnetic Janssen effect" $[8,9]$ and, as a result, the possibility to control the apparent mass of a ferromagnetic granular column. Therefore, as a follow-up of those studies, we propose here to go beyond the simple static configuration and investigate now the discharge of a silo filled with ferromagnetic particles. When submitted to an external magnetic field,

*e-mail: louison.thorens@ens-lyon.fr

A video is available at https://doi.org/10.48448/ejwj-jr49 each particle acquire a magnetic moment $\mathbf{d}$ leading to tunable magnetic pair interactions inside the grain assembly depending on the magnetic field direction and amplitude. The flow of such magnetic powder has been investigated previously in the case of a 3D funnel [10], with the report of an intermittent flow at the output of the funnel and a strong dependence of the flow rate on the magnetic field amplitude. Nevertheless, the 3D geometry introduces a complex network of ferromagnetic interactions. Here, we propose to focus on a $2 \mathrm{D}$ geometry for which the ferromagnetic interactions are reduced to pure repulsion. Previous work using permanent magnets in a $2 \mathrm{D}$ silo have been reported [11], however in this case the magnetic interactions cannot be tuned.

\section{Flow rate control}

A sketch of our experimental setup is given in figure 1a. The setup consists of a Hele-Shaw cell with a gap $e=$ $0.6 \mathrm{~mm}$ filled with $2 a=500 \mu \mathrm{m}$ diameter steel beads. The gap consists of two chambers separated by a $w=10 a$ wide opening formed by a $45^{\circ}$ funnel. This system is quasi $2 \mathrm{D}$ and the beads are arranged in a unique layer packing flowing from the top chamber to the bottom one by gravity. The system is placed in a magnetic coil wider than the cell generating a magnetic field $\mathbf{B}$ perpendicular to the cell plane. To quantify the strength of the magnetic interactions we consider the magnetic Bond number $[10,12]$ which compares the magnetic interactions and gravity $\Psi=\left(\chi_{m} B\right)^{2} /\left(\mu_{0} a \rho g\right)$ where $\chi_{m}$ is the magnetic susceptibility equals to 3 in our case [13], $\mu_{0}$ is the magnetic permeability of vacuum. In our experimental set-up, we could reach a maximum value of $\Psi=62$, corresponding to a magnetic field of $130 \mathrm{G}$.

Since the magnetic field is homogeneous in the cell plane, each particle $i$ has a magnetic dipolar moment $\mathbf{d}=\chi_{m} \mathbf{B} / \mu_{0}$ 
(a)

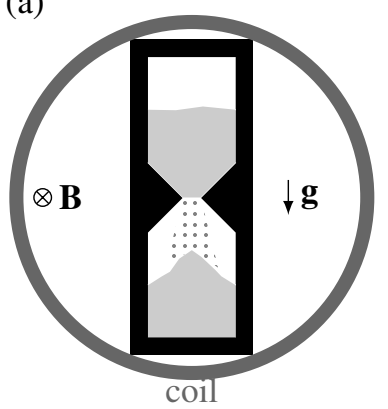

(b)

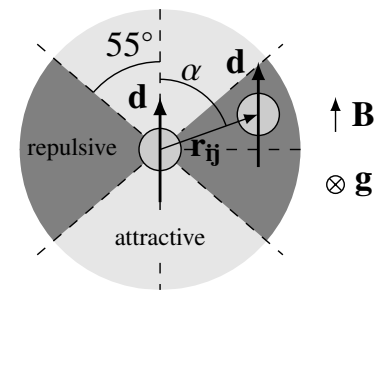

Figure 1. (a) Experimental setup made of a quasi 2D silo in a Hele-Shaw cell with a $45^{\circ}$ funnel in its centre. The cell is filled with $500 \mu \mathrm{m}$ diameter steel beads. The cell is placed in a magnetic coil generating a magnetic field perpendicular to the cell. (b) Top view of the left panel representing the dipolar interaction between two uniformly charged ferromagnetic beads characterized by the dipolar moment $\mathbf{d}$.

and interacts with its neighbour $j$ according to the magnetic potential energy:

$$
U_{i j}=\frac{\mu_{0} d^{2}}{4 \pi r_{i j}^{3}}\left(1-3 \cos ^{2} \alpha\right),
$$

where $r_{i j}$ is the beads distance and $\alpha$ is the angle between $\mathbf{r}_{i j}$ and the magnetic field $\mathbf{B}$. The magnetic pair interaction is summarized in figure 1b. In general, the interaction can be either repulsive or attractive depending on the angle $\alpha$, but in our case the quasi $2 \mathrm{D}$ geometry leads to a minimum angle $\alpha=\pi / 2-(\arcsin e / 2 a-1) \approx 78^{\circ}$ which means that the interactions are always repulsive.

The experimental protocol is the following: first the beads are at rest in the bottom chamber and we turn the magnetic field on. Then, the cell is rotated using a stepper motor to the next vertical position where the ferromagnetic particles are now in the upper chamber. A high-speed camera (Phantom V10) then records the discharge of the cell at a rate of 440 frames per seconds. Finally, the cell is rotated again and we perform a demagnetisation of the beads using the hysteresis protocol proposed in [18].

We estimated the flow rate using the evolution of the number of beads in the bottom chamber. This number was computed using the apparent area of the bead sedimented cluster occupying the lower chamber after a binarization of the image. Since the contrast between the black grains and the white background is high, the image analysis is rather straightforward and the detection robust. The temporal evolution of the cumulative number $\mathrm{N}(\mathrm{t})$ of those sedimented particles during the discharge of the silo is given in figure $2 \mathrm{a}$ for different amplitude of the applied magnetic field. We can see that the flow rate given by the slope of those curves $N(t)$ averaged over 5 different experimental realisations, displayed in figure $2 b$, decreases systematically when we increase the magnetic interaction intensity $\Psi$. This result was previously reported by [10] in the case of a 3D funnel where the magnetic dipole-dipole interactions within the granular packing could be either at-

(a)

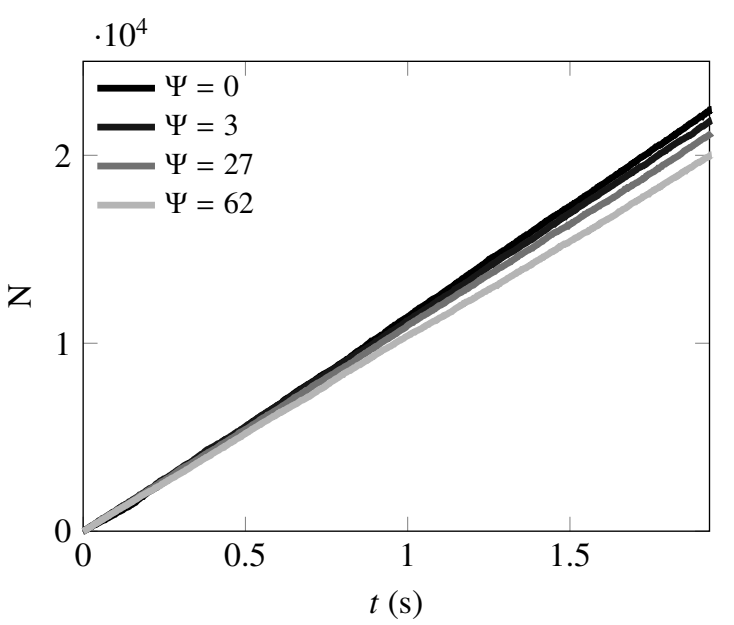

(b)

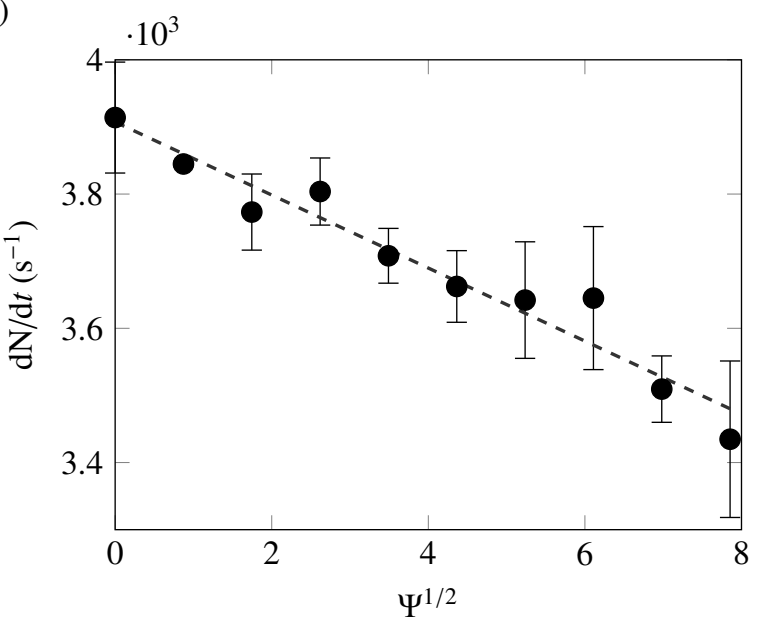

Figure 2. (a) Cumulative number of beads in the lower part of the cell during time for different magnetic field. (b) Flow rate of the beads in the silo given by the slope of the cumulative number of beads. The flow rate decreases when increasing the magnetic field intensity. The dashed line corresponds to a linear fit of the experimental data. Error bars correspond to the standard deviation over three realizations.

tractive or repulsive. In our case where we force the interactions to be purely repulsive, we retrieve this behaviour where the flow rate of the beads flowing through a narrow opening is controlled by the amplitude of the external magnetic field. Nevertheless, we did not find in this case, and for our reachable $\Psi$, a critical magnetic field threshold above which the bead do not flow anymore.

The measured flow rate seems to decrease linearly with the amplitude of the magnetic field $B \equiv \Psi^{1 / 2}$, as shown in figure $2 \mathrm{~b}$. This result appears different from the experimental measurement reported in [10], where the flow rate was found proportional to $\Psi$. This discrepancy could be explained by the different geometry studied (2D versus $3 \mathrm{D})$, involving different magnetic interactions within the granular packing.

\section{Internal heterogeneous dynamics}

Interestingly, the internal dynamics of the flowing grains within the upper chamber of the silo displays a more 


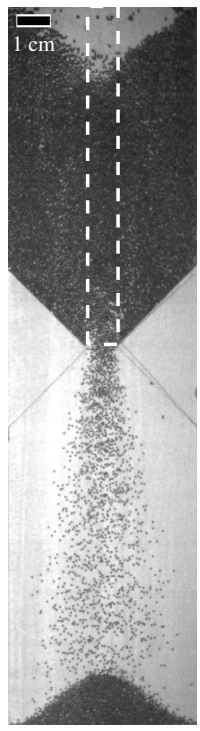

$t=0 \mathrm{~ms}$

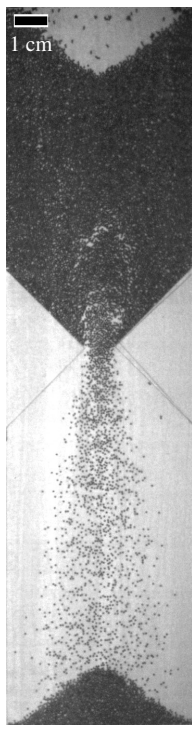

$t=33 \mathrm{~ms}$

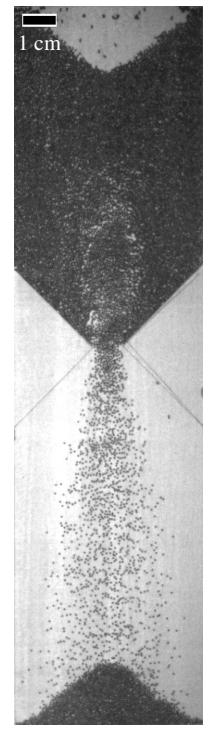

$t=67 \mathrm{~ms}$

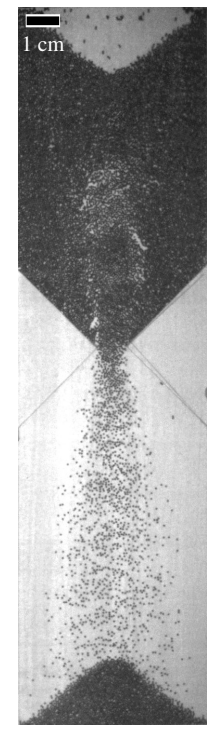

$t=100 \mathrm{~ms}$

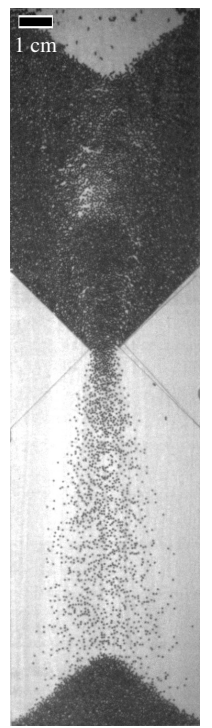

$t=130 \mathrm{~ms}$

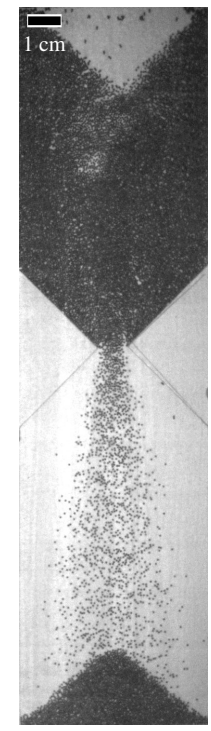

$t=170 \mathrm{~ms}$

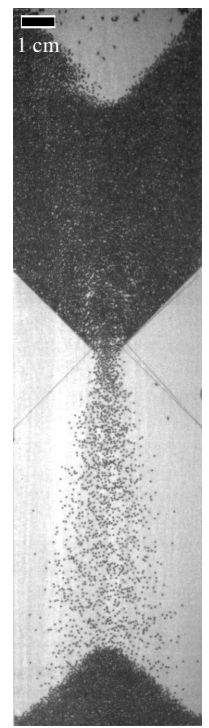

$t=200 \mathrm{~ms}$

Figure 3. Image sequence of a silo discharge in the case of the maximum magnetic field intensity $(\Psi=62)$. The images were taken at regular time steps and we can see a defect wave, where the beads are free falling inside the structure, going upward. The full sequence of $200 \mathrm{~ms}$ corresponds to a propagation cycle where the defect emerges at the funnel neck and go up to the free surface of the structure. The white dashed window shown in the first panel represents the window used for the spatio-temporal maps displayed in figure 4.

complex behaviour. Indeed, we could notice some strong spatio-temporal fluctuations of the density of the packing, which appeared more evidently when increasing the amplitude of the applied magnetic field. In figure 3, we show some typical examples of the flowing patterns on a series of images recorded during one experiment performed with the highest magnetic field $\Psi=62$ on a $200 \mathrm{~ms}$ time range. Examining the top chamber of the cell, we can notice the nucleation of a kind of air bubble, just at the bottom of the funnel, corresponding to a zone where particles are missing. Then, we observe that this bubble is moving upward within the packing, against the granular flow, until it reaches the top free surface in about $170 \mathrm{~ms}$. Furthermore, strikingly, the particles at the free surface of the packing do not move until this bubble could reach the surface; at that point, they fall abruptly, while a new bubble emerges in the opening area. Such heterogeneous granular flow is reminiscent of the early work of [14] reporting experiments of a ticking hourglass. In this case, the lower room is sealed and the flow rate is stopped by the air pressure until Darcy air flow through the grains unclogs the system. A ticking behaviour was also reported by [10] at the output of the funnel using magnetic interactions. Nevertheless, in our case the output flow rate remains constant, as shown in figure $2 \mathrm{a}$, while the heterogeneous dynamics appears only in the top chamber. Our results remind us more of the work presented in $[15,16]$, respectively for rough and cohesive grains. The observations that were made are close to ours where lower density waves move in the packing creating discrete avalanches of the grains free surface. The proposed explanation is that a part of the grains is clogging the rest of the packing, creating a low density beneath, until the cohesion is not strong enough to support the whole packing. The main advantage of our experiment is the easily tunable aspect of ferromagnetic interactions.

To quantify such complex dynamics, we computed spatio-temporal maps of the flowing particles. In practice, for each experiment, we considered a window of $10 a$ width and as high as the top part of the cell centred in the horizontal direction as shown in figure 3 on the first panel. At each time step, we average over the grey scale level of this region, along the horizontal direction (over 37 pixels) to obtain one single vector, giving the mean intensity detected along the vertical position in the chamber (a white zone corresponding to air, while the particles appear in black). The final picture gives a spatio-temporal evolution of the flowing grain packing in the top chamber as used in [17]. The results for three experiments are given in figure 4 . Without any magnetic field, $\Psi=0$, the grains flow out of the top part rather smoothly and the free surface of the packing goes down at a constant flow rate. For an intermediate magnetic field amplitude, $\Psi=27$, the flow rate remains constant with a linear evolution of the free surface of the packing. However we can distinguish the apparition of irregularities (whitish lines and a slightly rough top-interface) corresponding to the nucleation and motion of the bubbles, previously discussed. Moreover, the duration of the discharge is longer, corresponding to a lower flow rate as we reported in figure 2. Finally, for high magnetic field, $\Psi=62$, the discharge dynamics within the upper chamber of the cell appears to be very jerky. As we reported on the snapshots of figure 3 , and as it clearly emerges in the spatio-temporal figure, the free surface of the packing displays a step-like evolution. Such "stop and go" jerky dynamics is characterized by successive sudden 

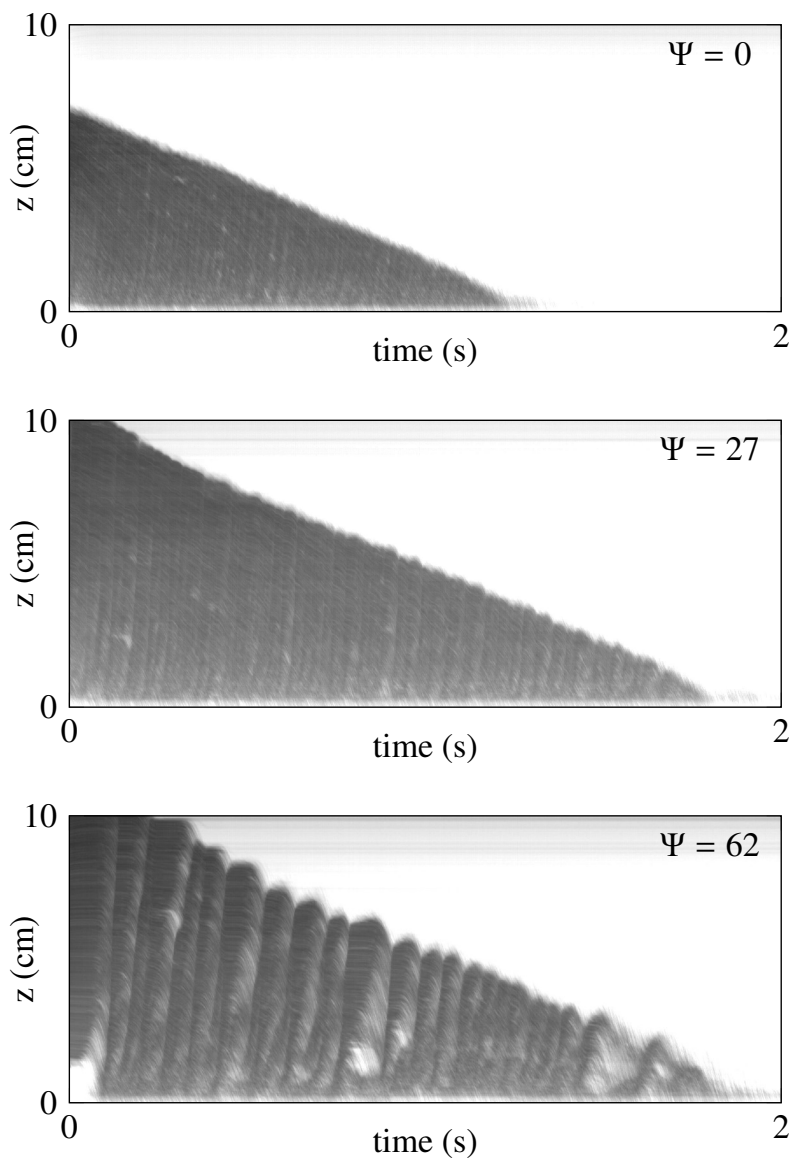

Figure 4. Spatio temporal maps of the flowing particles in the upper part of the silo structure for different magnetic field intensity $\Psi$. At each time step the vertical data corresponds to an average of the grey scale values over a $10 a$ width rectangle centred on the funnel displayed in the first image of figure 3. For low and medium magnetic field no wave can be seen but we retrieve that the flow rate, given by the free surface slope, decreases. For higher magnetic field, wave propagation given by the step structure in the image appears.

drops of the free surface, followed by waiting times during which the free surface more or less does not evolve. The characteristic pattern formed by the apparition of parallel white lines in the spatio-temporal map, corresponds indeed to the upward motion of a bubble - density defect within the packing.

The waiting time between two successive drops of the free surface corresponds to the time for the bubble-defect, nucleated at the funnel, to propagate through the packing and reach the free surface. Since the white lines in the spatio-temporal maps appear parallel, the velocity of those density-defects is constant and thus the waiting time decreases during an experiment. We can also notice that the white lines are steeper for a lower $\Psi$. Interestingly, one can also notice that the bubble density-defect velocity depends on the magnetic field amplitude. Moreover, the sudden drops observed at the free surface of about $2 \mathrm{~mm}$ of amplitude corresponds to an abrupt avalanche triggered when a bubble reaches the top free surface.

To conclude, we have shown that the discharge dynamics of a ferromagnetic grain assembly depends strongly on the external magnetic field applied. Interestingly, we could somehow realize a tunable hourglass, since the constant flow rate decreases systematically when increasing the amplitude of the applied external magnetic field. Moreover, for high magnetic field amplitudes, the flowing dynamics of the granular packing appears very heterogeneous, with the nucleation of a bubble at the funnel opening and rising through the particles assembly.

\section{References}

[1] H.M. Jaeger, S.R. Nagel, Science 255, 1523 (1992)

[2] H.M. Jaeger, S.R. Nagel, R.P. Behringer, Rev. Mod. Phys 68, 1259 (1996)

[3] P.G. de Gennes, Rev. Mod. Phys. 71, S374 (1999)

[4] W. Beverloo, H. Leniger, J. van de Velde, Chem. Eng. Sci. 15, 260 (1961)

[5] M.A. Aguirre, J.G. Grande, A. Calvo, L.A. Pugnaloni, J.C. Géminard, Phys. Rev. Lett. 104, 238002 (2010)

[6] C. Perge, M.A. Aguirre, P.A. Gago, L.A. Pugnaloni, D.L. Tourneau, J.C. Géminard, Phys. Rev. E 85, 021303 (2012)

[7] S. Rubio-Largo, A. Janda, D. Maza, I. Zuriguel, R. Hidalgo, Phys. Rev. Lett. 114, 238002 (2015)

[8] L. Thorens, K.J. Måløy, M. Bourgoin, S. Santucci, Nat. Commun. in press (2021)

[9] L. Thorens, K.J. Måløy, M. Bourgoin, S. Santucci, EPJ Web Conf. this volume (2021)

[10] G. Lumay, N. Vandewalle, Phys. Rev. E 78, 061302 (2008)

[11] G. Lumay, J. Schockmel, D. Henández-Enríquez, S. Dorbolo, N. Vandewalle, F. Pacheco-Vázquez, Papers in Physics 7, 070013 (2015)

[12] J. Vessaire, N. Plihon, R. Volk, M. Bourgoin, Phys. Rev. E 102, 023101 (2020)

[13] K. Bai, J. Casara, A. Nair-Kanneganti, A. Wahl, F. Carle, E. Brown, J. Appl. Phys. 124, 123901 (2018)

[14] T.L. Pennec, K.J. Måløy, A. Hansen, M. Ammi, D. Bideau, X. lun Wu, Phys. Rev. E 53, 2257 (1996)

[15] G.W. Baxter, R.P. Behringer, T. Fagert, G.A. Johnson, Phys. Rev. Lett. 62, 2825 (1989)

[16] E. Freyssingeas, M.-J. Dalbe, J.-C. Géminard, Phys. Rev. E 83, 051307 (2011)

[17] E. Mersch, G. Lumay, F. Boschini, N. Vandewalle, Phys. Rev. E 81, 041309 (2010)

[18] N.A. Spaldin, Magnetic Materials (Cambridge University Press, 2009), 18 